\title{
Gute Arbeit
}

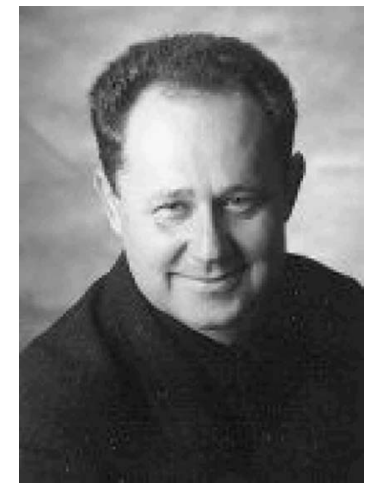

Prof. Dr. Med. Manfred Wildner
Bibliografie

DOI http://dx.doi.org/ 10.1055/s-0034-1398511 Gesundheitswesen 2015; 77: 6-7

(c) Georg Thieme Verlag KG Stuttgart · New York ISSN 0941-3790

Korrespondenzadresse Prof. Dr. med. Manfred Wildner

Bayerisches Landesamt für Gesundheit und Lebensmittelsicherheit

Veterinärstraße 2

85764 Oberschleißheim

manfred.wildner@lgl.bayern.de
Der französische Schriftsteller Andre Gide fragte einmal einen Chinesen, was ihn auf seinen Reisen am meisten beeindruckt hätte. Dieser erwiderte darauf, „dass er in Europa hauptsächlich den Ausdruck von Müdigkeit, Trauer und Sorge auf allen Gesichtern empfunden habe, wir kennten alle Künste, mit Ausnahme der so einfachen, glücklich $z u$ sein. Dass die Religion, die den Menschen sagt [...], sich gegenseitig zu helfen, einander zu lieben und dem die rechte Wange zu bieten, der einen auf die linke schlägt - gerade die unruhigsten, reichsten, listigsten, bewegtesten Völker gebildet hat, [...] die Völker schließlich, deren Ehre am kitzligsten ist und sich am meisten der Verzeihung und dem Ausgleich widersetzt: das konnte er nicht begreifen“ [1]. In wieweit Religionen in modernen Gesellschaften heute noch das Leitmotiv setzen, ist eine eigene Frage. Eine religiös mit bedingte Prägung besteht zweifelsohne in den verschiedenen Kulturkreisen. Unverkennbar ist darüber hinaus eine weltweit einheitliche zivilisatorische Überformung westlicher Prägung. Am deutlichsten wird dies in den homogenen städtischen Lebensformen der globalisierten „Megacities“ von Bombay bis Bangkok, London, Sao Paulo oder Tokio. Kennzeichen dafür sind auch die oben angesprochene listenreiche Konsumorientierung, das Streben nach Reichtum sowie die hohe Mobilität. Hier sei die Frage nach dem Zweck für dieses unruhige Tätigsein gestellt - vielleicht auch die oft ausgeklammerte Frage, welchem übergeordneten (Lebens-)Sinn es dient [2]. Naheliegend ist im Sinne der Maslowschen Bedürfnispyramide die Befriedigung der lebensnotwendigen Grundbedürfnisse Ernährung, Kleidung, geschützter Wohnraum [3]. Darauf aufsetzend finden sich Bestrebungen um soziale Anerkennung und Teilhabe am öffentlichen Leben, an Bildungschancen und an den kulturell-zivilisatorischen Angeboten. Dies schließt die Chancen von Selbstfindung und -entwicklung und damit verbundene höhere Aspirationen mit ein. Spätestens hier berühren sich die Fragen von Lebenszweck und Lebenssinn. Urbanes Leben mit seinen vielfältigen Arbeits-und Teilhabemöglichkeiten bietet augenscheinlich für viele Menschen die besseren $\mathrm{Zu}-$ gänge zu diesen Chancen. Ein Großteil dieses Lebens findet in organisierten Arbeitswelten statt: bei der Produktion von Gütern wie auch in Form von Dienstleistungen, in Werkstätten und in Büros, immer eingebunden in vielfältige und intensive Kommunikationsprozesse.

Diese modernen Arbeitsformen haben ihren Ausgang in den westlichen Industriegesellschaften und Marktwirtschaften genommen und sich über die globalisierten Handelswege und Märkte ausgebreitet. Was uns Märkte auch lehren, ist, dass die Dinge ihren Preis haben. Welchen Preis bezahlen wir für die geschilderten gesellschaftlichen Entwicklungen? Sind wir uns der Kosten, insbesondere auch der externalisierten gesellschaftlichen und unweltbezogenen Kosten, überhaupt ausreichend bewusst? Beispiele für solche nach außen verlagerten Kosten sind die Belastung von natürlichen Ressourcen wie Wasser, Boden und Luft - aber auch der Verschleiß der Gesundheit der Beschäftigten. Diese Kosten werden häufig der Allgemeinheit aufgebürdet und bilden sich nur unzureichend in den Marktpreisen ab wenn überhaupt. Ein Beispiel dafür sind die Umweltemissionen in China, eine zunehmend bedrohliche Kehrseite der erstaunlichen jährlich 2-stelligen wirtschaftlichen Wachstumsraten. So werden die gesundheitlichen Grenzwerte in der Außenluft in ganz Mittelchina - nicht nur in einzelnen Städten - dauerhaft um ein Vielfaches überschritten (www.aqicn.org). Die Bodenbelastung führt dort über die Schadstoffaufnahme durch Pflanzen und Tiere inzwischen zur Belastung der daraus erstellten Handelsprodukte wie Lederwaren und Textilien mit resultierenden Absatzproblemen auf den gesundheits- und umweltbewussteren westlichen Handelsmärkten. Die Wasserbelastung betrifft nicht nur die Ökosysteme von Seen, Flüssen und Meeren, sondern zunehmend auch den Verbrauch begrenzter Grundwasservorräte.

Sind derartige Produkte und Produktionsmethoden noch „gute Arbeit“? Auf die geschichtliche Möglichkeit des Zusammenbruchs ganzer Gesellschaften in enger Verflechtung mit dem Niedergang der natürlichen Lebensgrundlagen hat der amerikanische Ökologe Jared Diamond nachdrücklich hingewiesen, wie vor ihm bereits Thomas Robert Malthus oder der Club of Rome [4-6]. Wir können hier getrost - oder auch weniger getrost - von einer globalen Herausforderung sprechen: Die Sachstandsberichte des Weltklimarates sprechen eine deutliche Sprache (www.de-ipcc. de). Worauf Diamond auch hinweist, sind die auch gesellschaftlich fatalen Folgen ökologisch vermittelter wirtschaftlicher Krisen. Diese haben in der Vergangenheit zu einer Zunahme kriegerischer Auseinandersetzungen um noch verbliebene essentielle Ressourcen bis hin zu Völkermord, Kannibalismus und dem dennoch einsetzenden Aussterben der betroffenen „,siegreichen“ Gesellschaften geführt. Nur ein Relikt aus ferner Vergangenheit? Sicherlich nicht, wie der Völkermord in Ruanda noch Ende des 20. Jahrhunderts gezeigt hat. Weitere Beispiele lassen sich mühelos anfügen und sich mit Bezug zu Anbauflächen, 
Erdölvorkommen und zukünftig wohl auch Wasservorräten ohne allzu großen Aufwand für die Zukunft projektierten.

Um hier einem verkürzten Problemaufriss vorzubeugen: der Mensch „lebt nicht vom Brot allein“ (Matthäus 4,4). Auf vielfältige, subtile Weisen finden gesellschaftlich vermittelte Wirkungsketten ihren Weg in unsere heutigen Lebenswelten. So lässt sich fragen, ob eine Arbeitswelt noch gute Arbeit bietet, wenn sie von harschen Kommunikationsstilen, ständig engerer Terminsetzung, Arbeitsverdichtung und all gegenwärtigem Controlling, von Erreichbarkeit auch außerhalb der regulären Arbeitszeit und hoher Mobilität des Arbeitnehmers gemäß dem Idealbild des „flexiblen Menschen“ der Globalisierung (Richard Sennett) geprägt wird. Humaner scheinen differenziertere Ansätze wie die einer „Double Helix of Learning and Work“, welche eine kontinuierliche Abfolge von modularisierten Zeiten des Lernens und des Arbeitens bis in ein so ermöglichtes höheres Renteneintrittsalter vorsehen [7].

Schon früh wurde u.a. vom Deutschen Gewerkschaftsbund in den 1970er und 1980er Jahren die Humanisierung der Arbeitswelt thematisiert. Das auch darauf aufbauende gewerkschaftlich erarbeitete und vertretene Konzept einer Guten Arbeit knüpft an diese Bemühungen an sowie an das Leitbild von Decent Work der Internationalen Arbeitsorganisation (ILO). Es stellt das gewerkschaftliche Zukunftskonzept in Deutschland dar [8]. Eine ähnliche Zielstellung verfolgt seit 2002 die Initiative Neue Qualität in der Arbeit (INQA) des Bundesarbeitsministeriums unter Beteiligung von Arbeitnehmer- und Arbeitgebervertretungen, Sozialversicherungsträgern, Bund und Ländern. Diese will unter anderem die unternehmerischen Ziele mit den persönlichen sozialen Interessen und den gesellschaftlichen sozialen Zielen harmonisieren. Von der Europäischen Union wurde Gute Arbeit in die „Gemeinschaftsstrategie zu Gesundheit und Sicherheit bei der Arbeit für den Zeitraum 2007-2012“ aufgenommen.

Ein mehrfacher Gesundheitsbezug ist für Humanität und Qualität in der Arbeit gegeben. Er betrifft gesundheitsgefährdende physikalisch-chemische Arbeitsbedingungen genauso wie die Auslaugung der mentalen menschlichen Ressourcen. Depressionen einschließlich des „Burn out“ sind zur führenden Ursache von Frühberentungen in Deutschland geworden und führen zu einem stetig steigenden Bedarf an entsprechenden Behandlungs- und Rehabilitationskapazitäten. Nicht zu übersehen ist auch, dass das bisher geschützte Privatleben und die dort lebenden engen Bezugspersonen durch die geschilderten Entgrenzungen der Berufswelt zunehmend in diese Entwicklungen mit einbezogen werden: durch Heimarbeitsplätze, eine ständige Erreichbarkeit, Mehrarbeit und beruflich geforderte Reisetätigkeiten [9].

Diese Entwicklungen haben auch das Gesundheitswesen erreicht, mit allen beschriebenen Facetten der Intensivierung der Prozesse und Entgrenzung der Arbeitswelt. Hinzu kommen neue Aktivitäten, welche dem Erhalt bzw. der Steigerung der Arbeitsfähigkeit dienen sollen: von einem ethisch wenig problematischen zweiten Gesundheitsmarkt mit Schwerpunkt Wellness über Angebote der medikamentösen Leistungssteigerung bis zum Einfrieren von Eizellen im Dienst der Verfügbarhaltung weiblicher Arbeitskraft. Die Frage nach „Guter Arbeit“ im Gesundheitsbereich betrifft damit zum einen die Arbeits- und Ausbildungsqualität für die im Gesundheitswesen Beschäftigten, zum anderen die Inhalte und die Ergebnisqualität der vielfältigen gesundheitlichen Dienstleistungen.

Diesen und weiteren Fragen gehen die Beiträge in diesem Heft nach, mit speziellen Themen wie Rationierungen im deutschen Gesundheitswesen insbesondere in der Onkologie, hausärztlichen Arzneimittelpriorisierungen bei stationär entlassenen multimorbiden Patienten, die flächendeckende Erreichbarkeit von Organkrebszentren, Einflussfaktoren auf Erlöse und Kosten der Krankenhäuser, der Dienstleistungsproduktivität in der Krankenhauspflege, der altersbezogenen mentalen Leistungsfähigkeit bei Lehrerinnen, dem FIMA-Fragebogen zu Gesundheitsleistungen im Alter sowie der Entwicklung von standardisierten Bewertungssätzen für gesundheitsökonomische Evaluationen. Wenn unser zitierter chinesischer Reisender den Ausdruck von Müdigkeit, Trauer und Sorge auf den Gesichtern in den europäischen Ländern beklagt hat, so mag dies seine Ursachen auch im Fehlen „guter Arbeit“ gehabt haben. Vielleicht würde er, darauf angesprochen, auf wichtige Werte aus seinem Kulturkreis hinweisen: Auf das Streben nach Ausgleich und gesellschaftlicher Harmonie sowie die Verbindung von universaler Weltverantwortung mit den konkreten Alltagspflichten, wie sie für den Konfuzianismus typisch sind, vielleicht auch auf die Konzilianz und Toleranz des Buddhismus, seine allgemeine Wesenliebe und mitfühlende Hilfsbereitschaft als Kennzeichen einer inneren Entwicklung zur Freiheit hin [1]. Hinweise, die auch einer allgemeinen Gesellschaftsethik zuzurechnen sind und sehr konkret auch guter Arbeit. Dabei ist, wie so oft, der Einzelne genauso anzusprechen wie das gemeinsam gestaltete Umfeld. Vielleicht gelänge so auch uns westlichen Menschen ein Lächeln, über das ein zukünftiger chinesischer Reisender sagen könnte: „Sie lächeln, weil sie [aus gutem Grund] lächeln können!“ [10]. In diesem Sinne uns allen ein gutes Neues Jahr!

\section{Literatur}

1 von Glasenapp $H$. Die fünf Weltreligionen: Hinduismus, Buddhismus, Chinesischer Universismus, Christentum, Islam. München: Hugendubel, 1963, 2001 (Sonderausgabe); 435-436

2 Hannah Arendt: Vita activa oder vom tätigen Leben. München, Zürich: Piper, 2002

3 Maslow AH. Motivation and personality. Harper \& Row, New York: 1954 überarbeitete Ausgabe 1970

4 Diamond J. Kollaps: Warum Gesellschaften überleben oder untergehen. Frankfurt a.M.: Fischer Verlag, 2006 engl. Original: New York, Viking 2005

5 Malthus TR. Das Bevölkerungsgesetz. München: dtv-Taschenbuch, 1977 (Originaltitel: An essay on the principle of population as it affects the future improvement of society, with remarks on the speculations of Mr. Godwin, M. Condorcet, and other writers, übersetzt von Christian M. Barth)

6 Meadows DH, Meadows DL, Randers J et al. Die Grenzen des Wachstums. Bericht des Club of Rome zur Lage der Menschheit. Aus dem Amerikanischen von Hans-Dieter Heck. Stuttgart: Deutsche Verlags-Anstalt, 1972

7 Giarini O, Malitza M. The Double Helix of Learning and Work. Bukarest: UNESCO-CEPES Studies on Science and Culture, 2003

8 Schröder L, Urban H-J, Hrsg. Gute Arbeit: Handlungsfelder für Betriebe, Politik und Gewerkschaften (Jahrbuch). Frankfurt a. M.: Bund, 2009

9 Kuhn J. Gute Arbeit als Leitbild der arbeitsweltbezogenen Gesundheitspolitik. In: Giesert M, Hrsg. Prävention: Pflicht \& Kür - Gesundheitsförderung und Prävention in der betrieblichen Praxis. Hamburg: VSA-Verlag, 2008

10 Hart $W$. Vipassana Meditation. The Art of Living as taught by S.N. Goenka. New York: Harper \& Row, 1987 Seite 127 